\title{
Um Modelo Explicativo para o Bem-Estar Subjetivo: Estudo com Mestrandos e Doutorandos no Brasil
}

\author{
An Explanatory Model for Subjective Well-Being: A Study with Masters \\ and PhD Students in Brazil
}

\author{
André Faro* \\ Universidade Federal de Sergipe, São Cristóvão, Sergipe, Brasil
}

\begin{abstract}
Resumo
O presente estudo teve como principal intuito mapear as relações entre estressores, estresse, enfrentamento e bem-estar subjetivo em mestrandos e doutorandos no Brasil, tendo sido elaborado um modelo explicativo da determinação do bem-estar subjetivo nessa população. Para tanto, participaram 2150 pós-graduandos no Brasil, entre mestrandos e doutorandos, oriundos das cinco regiões do país e de todas as grandes áreas do conhecimento da Coordenação de Aperfeiçoamento de Pessoal de Nivel Superior (CAPES). Os instrumentos utilizados foram um questionário sobre estressores na pós-graduação, a Escala de Estresse Percebido, a Escala de Modos de Enfrentamento de Problemas e a Escala de Bem-Estar Subjetivo. Ao final, constatou-se que o modelo do bem-estar subjetivo, testado através de path analysis, denotou satisfatório ajuste e corroborou o pressuposto teórico.

Palavras-chave: Bem-estar subjetivo, estratégias de enfrentamento, estresse, estressores, pósgraduação.
\end{abstract}

\begin{abstract}
The main objective of the current study is to map the relationships among stressors, stress, coping, and subjective well-being in Master's and PhD students in Brazil. It has also been drawn up an explanatory model of the determination of subjective well-being in the mentioned population. Participants were 2150 Master's and PhD students in Brazil, from the five regions of the country and from all major knowledge areas of CAPES (Coordenação de Aperfeiçoamento de Pessoal de Nivel Superior). The instruments used were a questionnaire about stressors in post-graduate programs, the Perceived Stress Scale, the Ways of Coping Scale, and the Scale of Subjective Well-Being. Finally, it was found that the model of subjective well-being, tested by path analysis, denoted satisfactory goodness-of-fit indices and corroborated the theoretical assumption.

Keywords: Subjective well-being, coping strategies, stress, stressors, post-graduation.
\end{abstract}

Especialmente nos últimos dez anos, o Brasil vem assumindo, de modo progressivo, lugar de destaque no cenário científico internacional (Ministério da Ciência e Tecnologia [MCT], 2010). No compasso da ascensão econômica e social ocorrida nas últimas décadas, a produção científica brasileira e a formação de pesquisadores encontram-se em pleno crescimento, exibindo saltos quantitativos (ex. registro de patentes e volume de publicação em importantes periódicos) e qualitativos (ex. avaliação dos programas de pós-graduação e internacionalização dos cursos) que reiteram a importância do investimento

\footnotetext{
*Endereço para correspondência: Avenida Sílvio Teixeira, 691, Ed. Horto do Ipê, Apto. 1402, Aracaju, SE, Brasil 49025-100. E-mail: andrefaro@superig.com.br Pesquisa financiada pela Fundação de Amparo à Pesquisa e à Inovação Tecnológica do Estado de Sergipe (FAPITEC-SE) e Conselho Nacional de Desenvolvimento Científico e Tecnológico (CNPq)
}

em ciência e tecnologia (Ministério da Educação [ME] \& Coordenação de Aperfeiçoamento de Pessoal de Nível Superior [CAPES], 2010).

As políticas voltadas ao aperfeiçoamento do sistema de pós-graduação stricto sensu, composto basicamente pelos cursos de mestrado (acadêmico e profissional) e doutorado, mostram-se prioritárias para a escalada científica brasileira, pois a expansão e qualificação desse sistema impacta diretamente na geração de riquezas, competitividade internacional e desenvolvimento interno do país (Centro de Gestão e Estudos Estratégicos [CGEE], 2010, 2011; ME \& CAPES, 2010). Porém, a despeito dos avanços institucionais alcançados, principalmente no que tange a maior quantidade de cursos de mestrado e doutorado hoje disponibilizados nas universidades, a pós-graduação deve ser apreendida como uma realidade complexa, sobretudo, para o estudante de pós-graduação. Com efeito, a opção pela formação stricto sensu requer diversos ajustamentos 
em sua própria vida, desde um novo planejamento de carreira, aos desafios cotidianos de se cursar um mestrado ou doutorado.

A empreitada da pós-graduação produz inúmeras demandas para os estudantes, compreendendo tanto dificuldades objetivas (ex. prazos e metas), como pressões subjetivas (ex. competitividade e sucesso). Tais aspectos se configuram, usualmente, como elementos estressores que incidem sobre os pós-graduandos durante seu curso, podendo ser citadas situações ilustrativas como o tempo, frequentemente considerado escasso para a consecução do relatório final; a ausência de bolsas para todos os pós-graduandos e o próprio valor destas, geralmente aquém do desejável; a cobrança dos orientadores/coordenadores pelo ótimo desempenho dos alunos; e, por fim, o dilema "publicar ou perecer", que tende a sobrecarregar mestrandos e doutorandos, assim como os demais pesquisadores, em vista da necessidade de produzir cada vez mais e melhor (André, 2007; Aquino, 2008; Escobar \& Verdinelli, 2010; Horta, 2006; Morosini, 2009; Santos \& Alves, 2007; Verhine, 2008; Voltarelli, 2002).

Dado o exposto, entende-se que essas demandas estressógenas influenciam o ajustamento psicológico e psicossocial dos pós-graduandos, caracterizando a pós-graduação como um ambiente promotor de estresse (Santos \& Alves, 2007). O estresse atualmente é concebido como um fenômeno psicossocial que possui repercussão biológica, eliciado frente a uma ameaça real ou imaginada que seja percebida como capaz de afetar a integridade mental e/ou física do indivíduo (Santos, 2010). Quando há a exposição prolongada ao estressor, o estresse, além de impactar prejudicialmente sobre a saúde (Cohen, Janick-Deverts, \& Miller, 2007; Monroe, 2008), influencia negativamente o desempenho do estudante, o que afeta a qualidade de seu trabalho e da própria formação acadêmica (Dusselier, Dunn, Wang, Shelley, \& Whalen, 2005; Giancola, Grawitch, \& Borchet, 2009; Voltarelli, 2002).

A fim de entender como os estudantes lidam com esse estresse produzido na pós-graduação, vem à pauta os modos pelos quais eles enfrentam os desafios de sua formação. Sabe-se, sobre isso, que os indivíduos, quando em um contexto de estresse, recorrem a estratégias que visam a minimizar o impacto dos estressores sobre seu ajustamento, mobilizando recursos intra e extra-individuais para lidar com a situação eliciadora de estresse (Taylor \& Stanton, 2007). Logo, nesse enquadramento de manejo e confronto com os estressores, a teoria do enfrentamento ocupa hoje papel central na explicação dos mecanismos de adaptação frente ao estresse (Chang et al., 2007; Monroe, 2008; Santos, 2010).

Segundo Skinner e Zimmer-Gembeck (2007), o processo de enfrentamento se configura como ações regulatórias sob condições de estresse, o que envolve como as pessoas mobilizam, direcionam ou manejam comportamentos, emoções e pensamentos diante de situações estressoras, cujo efeito se percebe através da alteração do desfecho adaptativo. Desta feita, o conceito que alcançou maior expansão dentre as teorias que buscam explicar o estresse foi o de Lazarus e Folkman (1984), que entende o enfrentamento como constantes mudanças cognitivas e esforços comportamentais que tencionam manejar demandas externas e internas que são avaliadas como sobrecarrega ou excesso sobre a capacidade adaptativa do indivíduo. Devido a essa função, o enfrentamento é um mediador cognitivo do estresse, com o papel fundamental de combater os efeitos resultantes da percepção de estressores e encontrar formas de administrar o acúmulo de excitação provocado pela necessidade alteração do status adaptativo (Monroe, 2008; Santos, 2010; Taylor \& Stanton, 2007).

No processo de enfrentamento, duas estratégias primordiais foram concebidas por Lazarus e Folkman (1984): o Foco no Problema e o Foco na Emoção. A primeira se refere aos esforços empreendidos na tentativa de manejar ou solucionar a situação eliciadora do estresse, o que envolve, por exemplo, a busca por informações, decisões centradas no caráter do estressor, planejamento e busca ativa pela resolução de conflitos. A segunda focaliza a regulação das emoções derivadas da avaliação do estressor, objetivando administrar a repercussão emocional ocasionada pelo estímulo, como por exemplo, evitar confrontar-se com o estressor, distanciamento afetivo e a atenção seletiva.

Além dessas, atualmente se destacam outras duas formas de enfrentamento: o religioso e o suporte social. Sobre o enfrentamento religioso, entende-se que é o nível pelo qual a religiosidade está envolvida com o processo de manejo do estresse, indo além da pertença a uma filiação religiosa e revelando comportamentos e pensamentos que associam a administração do estresse às crenças em entidades superiores e a própria espiritualidade (Faria \& Seidl, 2005; Koenig, 2007). Já o suporte social, é basicamente apreendido como o tipo, quantidade, nível de integração e disponibilidade de relações de apoio e/ou suporte social percebido pelo indivíduo no momento de confronto com estressores (Seidl, Tróccoli, \& Zannon, 2001; Vangelist, 2009), o que usualmente atenua o impacto do estresse.

De modo geral, entende-se que o manejo do estresse, por meio do processo de enfrentamento, funciona enquanto mediador da percepção de Bem-Estar Subjetivo (BES), que é um reflexo da qualidade do ajustamento do indivíduo às diversas circunstâncias estressoras com as quais se depara (Antoniazzi, Dell'Aglio, \& Bandeira, 1998; Folkman \& Moskowitz, 2004). Logo, conhecer como os estudantes administram o estresse da pós-graduação pode ser uma via para entender variações no BES diante dos estressores do mestrado e doutorado.

O BES é tido como uma faceta da saúde geral (Galinha \& Ribeiro, 2005), ou mesmo, a felicidade (Costa \& Pereira, 2007). Em uma visão mais ampla, é descrito como a qualidade de vida percebida em um dado momento da história do indivíduo, o que vai além dos limites da saúde biológica ou mental, isto é, embora sob pressão ou condição de adoecimento, o indivíduo pode sentir-se feliz (Albuquerque \& Tróccoli, 2004; Diener, 2000; Siqueira \& 
Padovan, 2008). O BES geralmente é mensurado através do índice de satisfação com a vida (componente cognitivo) e a frequência de afetos negativos e positivos (componente afetivo) na experiência cotidiana, indicando a qualidade do ajustamento; se boa, com predomínio de afetos positivos e alta satisfação; ruim, com predomínio de afetos negativos e baixa satisfação, além de variações entre esses polos (Albuquerque \& Tróccoli, 2004; Costa \& Pereira, 2007; Siqueira \& Padovan, 2008).

Um nível de BES elevado reflete satisfatório ajustamento às demandas contextuais que o individuo vivencia (ex. bom desempenho, motivação, satisfação, etc.), o que tende a espelhar capacidade de obter prazer, e sentir-se produtivo, em suas atividades cotidianas (Dela Coleta \& Dela Coleta, 2006; Diener, 2000; Galinha \& Ribeiro, 2005; Siqueira \& Padovan, 2008). Portanto, a análise do BES em estudantes de pós-graduação pode favorecer a identificação do nível de adaptação dos pós-graduandos aos estressores que se lhes apresentam no decurso de sua formação, os quais evocam estado de estresse e, por isso, ativam o uso de estratégias de enfrentamento, alterando, ao final, o BES.

Dado o exposto, o presente estudo teve como principal intuito mapear as relações entre estressores, estresse, enfrentamento e BES em mestrandos e doutorandos no Brasil. Para tanto, foram estabelecidos os seguintes objetivos:

1. Identificar a distribuição dos principais estressores da pós-graduação stricto sensu;

2. Detectar o índice de estresse dos estudantes de mestrado e doutorado;

3. Evidenciar as estratégias de enfrentamento mais utilizadas;

4. Determinar o índice de BES desses estudantes e,

5. Testar um modelo explicativo para o BES dos pós-graduandos, tendo estressores, estresse e enfrentamento enquanto preditores.

\section{Método}

\section{Participantes}

A amostra foi composta por 2150 pós-graduandos stricto sensu no Brasil, sendo $61,8 \%$ mestrandos $(n=$ $1329)$ e $38,2 \%$ doutorandos $(n=821)$. No total, foram $29,1 \%$ do sexo masculino $(n=625)$ e $70,9 \%$ do feminino $(n=1525)$, com média de idade em 29,4 anos $(D P=7,20)$. Houve participantes de todo o país, exceto dos Estados do Piauí e Roraima. A proporção por regiões ficou em 3,3\% do Norte $(n=72), 8,9 \%$ do Centro-Oeste $(n=192), 17,4 \%$ do Nordeste $(n=374), 34,2 \%$ do Sul $(n=735)$ e $36,1 \%$ do Sudeste $(n=777)$.

Os participantes foram oriundos de 66 instituições e mais de 100 programas de pós-graduação. Quanto à vinculação dos programas, assim distribuíram-se: $39 \mathrm{em}$ universidades federais $(59,0 \%), 15$ estaduais $(22,7 \%), 9$ privadas $(13,6 \%)$ e 3 institutos de pesquisa $(4,7 \%)$. Por vínculo dos estudantes, foram $66,1 \%$ de universidades federais ( $n=1421), 28,4 \%$ de estaduais $(n=610), 3,4 \%$ de privadas $(n=74)$ e $2,1 \%$ de institutos de pesquisa $(n=45)$. De acordo as grandes áreas do conhecimento, participaram pós-graduandos da Linguística, Letras e Artes (3,1\%; $n=$ 67), Ciências Exatas e da Terra $(7,3 \% ; n=156)$, Engenharias $(9,4 \%, n=203)$, Ciências Agrárias $(10,0 \% ; n=214)$, Ciências Humanas $(12,8 \% ; n=276)$, Ciências da Saúde $(14,2 \% ; n=306)$, Ciências Sociais Aplicadas $(15,6 \% ; n=$ 336) e das Ciências Biológicas $(27,5 \% ; n=592)$.

A técnica de amostragem foi não probabilística, por conveniência e bola-de-neve. Os estudantes foram convidados via email encaminhado aos representantes de turma, listas de alunos ou coordenadores de pós-graduação, estando incluído o termo consentimento livre e esclarecido. Ao concordar com sua participação na pesquisa, o que ocorreu por meio do reenvio do questionário respondido ao email do pesquisador, foi solicitado aos estudantes que encaminhassem o convite da pesquisa para colegas mestrandos ou doutorandos no Brasil.

$\mathrm{O}$ projeto de pesquisa original, considerando que a presente investigação é continuidade de outro estudo (Santos \& Alves, 2007), foi aprovado pelo Comitê de Ética em Pesquisa com Seres Humanos da Universidade Federal de Sergipe (UFS), cujo protocolo é o de número 0085.0.107.000-05.

\section{Instrumentos}

Aplicou-se a Escala de Estresse Percebido (Perceived Stress Scale - PSS), traduzida e validada para o Brasil por Luft, Sanches, Mazo e Andrade (2007). A PSS é composta por 14 perguntas, com respostas em escala Likert de 5 pontos [de 0 (nunca) a 4 (sempre)] e escore final entre 0 e 56 pontos. Tal como procedido por Luft et al. (2007) e novamente efetuado para a presente validação, os itens ficaram equacionados em 7 negativos [Fator $1 \mathrm{com}$ alfa de Cronbach $(\alpha)=0,80]$ e 7 positivos (Fator $2 \operatorname{com} \alpha=$ $0,82)$. A confiabilidade $(\alpha)$ da escala total ficou em 0,88 .

Para os estressores, utilizou-se um questionário com 15 preocupações para com a pós-graduação. Nelas, os participantes deveriam responder o quanto aquelas situações lhes inquietavam em seu curso, sendo assinaladas em escala Likert de 5 pontos [ 0 (nada) a 4 (extremamente preocupado)]. A elaboração dessa lista de preocupações pautou-se nas orientações de Streiner e Norman (2008) quanto à construção de escalas. Assim, sua base foi inspirada na pesquisa de Santos e Alves (2007), que abordaram estressores percebidos por mestrandos em ciências da saúde, sendo complementada com debates extraídos de diversos estudos que mencionaram desafios vivenciados pelos pós-graduandos no Brasil.

A lista de preocupações foi encaminhada para a avaliação de 5 juízes (docentes de mestrado e/ou doutorado), a fim de verificar o quanto os itens atendiam aos seguintes critérios: (a) Clareza da assertiva, (b) Objetividade, (c) Pertinência ao objeto de estudo e (d) Relevância temática. A amplitude de resposta variou entre 1 (muito inadequado) a 5 (muito adequado) em cada quesito. Tendo sido efetuados ajustes na redação, conforme orientação dos juízes, 
a média geral de concordância alcançou 4,7, indicando satisfatória concordância.

A escala de preocupações foi submetida à análise fatorial exploratória (PAF, rotação oblíqua) e análise de confiabilidade dos fatores [Alfa de Cronbach $(\alpha)$ ]. Os índices alcançados $(\mathrm{KMO}=0,895$ e esfericidade de Bartlett $p<0,001)$ indicaram que a escala atendia ao critério de fatorabilidade, com elevada confiabilidade geral $(\alpha=$ 0,85 ). A melhor solução foi obtida com a extração de três fatores, abaixo discriminados:

1. Tempo e Recursos Financeiros $(\alpha=0,73)$ : $\mathrm{O}$ autovalor ficou em 5,144, com variância explicada de $30,7 \%$ e cargas fatoriais entre 0,475 e 0,892 . O fator 1 foi composto por 4 itens [2 (interferência da demanda dos estudos sobre outros aspectos de sua vida), 10 (questões financeiras relacionadas ao fato de estar estudando em tempo parcial ou integral), 11 (tempo para concluir a tese ou dissertação) e 12 (questões relativas ao calendário e prazos da pós-graduação)].

2. Demandas do Curso $(\alpha=0,66)$ : Autovalor em 1,346 , com variância explicada de $5,8 \%$ e cargas fatoriais entre 0,403 e 0,681 . O fator 2 ficou composto por 4 itens [ 3 (aproveitamento das disciplinas ofertadas), 8 (apresentações orais), 13 (questões relativas ao horário das aulas na pós-graduação) e 15 (possibilidade de notas inferiores as esperadas)].

3. Supervisão e Desempenho $(\alpha=0,76)$ : O autovalor encontrado foi de 1,143 , com variância explicada de 3,229 e cargas fatoriais entre 0,449 e 0,672 . O fator 3 ficou composto por 6 itens [1 (pressão interna pelo bom desempenho - cobrança pessoal elevada, otimização do desempenho, etc.), 4 (baixa quantidade de contatos com o orientador), 5 (dificuldade do tema escolhido), 6 (pressão externa acerca da conclusão - social, acadêmica, etc.), 7 (aproveitamento das supervisões) e 9 (possibilidade de não atingir o desempenho esperado pela banca)].

Destaca-se que o item 14 das preocupações (possível decepção quanto à inserção profissional) não se agregou a nenhum fator, sendo excluído da escala na modelagem final.

Analisou-se o enfrentamento por meio da Escala de Modos de Enfrentamento de Problemas (EMEP; Seidl et al., 2001). É uma escala composta por 45 itens em quatro fatores, todos com índices de confiabilidade satisfatórios na presente pesquisa: Foco no Problema (18 itens; $\alpha=$ 0,84), Foco na Emoção (15 itens; $\alpha=0,80$ ), Busca pela Religiosidade e/ou Pensamento Fantasioso (7 itens; $\alpha=$ 0,69 ) e Busca por Suporte Social (5 itens; $\alpha=0,78$ ). As questões são sentenças afirmativas e respostas dadas em uma escala de tipo Likert de 5 pontos [de 0 (eu nunca faço isso) a 4 (eu faço isso sempre)].

Aplicou-se, por fim, a escala de Bem-Estar Subjetivo (BES) de Albuquerque e Tróccoli (2004), composta originalmente por 62 itens, divididos em duas dimensões: a primeira como a experiência de afetos positivos e afetos negativos, respondidos em escala Likert de 5 pontos [0 (nem um pouco) a 4 (extremamente)], sendo 21 positivos e 26 negativos. A segunda dimensão versa sobre afirmativas quanto à satisfação com a vida (15 itens), respondida em escala de 5 pontos, variando desde 0 (discordo plenamente) a 4 (concordo plenamente). Refez-se a análise fatorial nos moldes da validação original e foi percebido que os fatores de agruparam do mesmo modo, todavia, havendo a exclusão do item 3 (amável) nos afetos positivos. A confiabilidade, na versão final, também foi satisfatória (afetos negativos, $\alpha=0,95$; afetos positivos, $\alpha=0,95$ e satisfação com a vida, $\alpha=0,91$ ).

\section{Análise dos Dados}

Inicialmente, conduziram-se procedimentos exploratórios e de ajuste no banco de dados com o programa SPSS (versão 19), em que se verificou a adequação ao modelo geral paramétrico (Tabachnick \& Fidell, 2007). A variável dependente (VD) do estudo foi o índice do BES, construído, para este estudo, através da soma das médias dos afetos negativos (AN), dos afetos positivos (AP) e da satisfação com a vida (SV), com o resultado do somatório dividido por $3(\mathrm{AN}+\mathrm{AP}+\mathrm{SV} / 3)$. Para os escores das escalas (PSS e fatores da EMEP) e do questionário de estressores (por fatores), calcularam-se média e desvio padrão.

As variáveis independentes (VI's) da análise foram, entre as sociodemográficas: sexo, idade, curso (se mestrando ou doutorando), região do país e área do conhecimento. Nos construtos, foram VI's: os fatores do questionário de estressores, as médias da PSS e dos fatores da EMEP. Nas análises bivariadas, foram aplicados os testes $T$ de Student (sexo e curso), ANOVA one-way e teste post-hoc Tukey (região e área do conhecimento), e correlação de Pearson (demais VI's), cujo nível de significância adotado foi de $p<0,05$.

Para mapeamento das relações entre os estressores, o estresse, o enfrentamento e o BES elaborou-se um modelo explicativo, que foi analisado através da técnica Path Analysis (Análise de Caminhos) e método o Maximum Likelihood (Máxima Verossimilhança), no software AMOS (versão 18). Na avaliação do ajuste do modelo (Byrne, 2010; Garson, 2003), utilizaram-se 4 condições: (a) Goodness of Fit Index (Índice de Bondade do Ajuste [GFI]) e (b) Comparative Fit Index (Índice de Comparação do Ajuste [CFI]) - para ambos, valores desejados acima de 0,90; (c) Expected Cross-Validation Index (Índice Esperado de Validação Cruzada [ECVI]), que serve para comparar modelos, sendo que o de menor valor é o mais apropriado; e (d) Root Mean Square Error of Aproximation (Raiz Quadrada da Média dos Quadrados dos Erros de Aproximação [RMSEA]), com valor desejado até 0,8 .

\section{Resultados}

Quanto aos estressores, a maior média foi a do fator Tempo e Recursos Financeiros $(M=2,4 ; D P=0,88)$, 
seguido dos fatores Supervisão e Desempenho $(M=1,6$; $D P=0,72)$ e Demandas do Curso $(M=1,2 ; D P=0,69)$. A média de estresse foi de 29,1 pontos $(D P=8,49)$, com extremos em 3 e 51 pontos. A estratégia de enfrentamento mais utilizada foi o Foco no Problema $(M=2,1 ; D P=$ $0,42)$, seguindo-se da Busca por Suporte Social $(M=1,9$; $D P=0,70)$, do Foco na Emoção $(M=1,8 ; D P=0,70)$ e da Busca pela Religiosidade e/ou Pensamento Fantasioso $(M=0,7 ; D P=0,51)$. Na escala de BES, a média da satisfação com a vida foi de 2,7 pontos $(D P=0,65)$, dos afetos positivos 2,1 $(D P=0,66)$ e dos negativos 1,7 (DP $=0,81)$. Logo, pelo cálculo do índice, a média do BES da amostra total foi de 2,2 pontos $(D P=0,32)$, com extremos em 0,7 e 3,4 . Para todas essas variáveis, a amplitude foi entre 0 e 4 pontos possíveis.

Ao se efetuar a comparação do BES, por sexo, não se constatou diferença estatisticamente significativa $[t(g l)=$ $-0,519(2148) ; p=0,604]$, com distribuição praticamente igual para o feminino $(M=2,2 ; D P=0,32)$ e masculino $(M=2,2 ; D P=0,33)$. Coincidentemente, na comparação por curso, as distribuições foram basicamente as mesmas: mestrandos com média 2,2 $(D P=0,32)$ e doutorandos 2,2 $(D P=0,33)$, não havendo significância estatística $[t(g l)=$ $1,394(2148) ; p=0,163]$. No caso da idade, aplicou-se a correlação de Pearson e também não se constatou significância $(r=-0,005 ; p=0,825)$.

$\mathrm{Na}$ análise do BES, segundo a região do país, não foi encontrada significância estatística $[F(4,2145)=0,862 ; p$ $=0,486$; teste de Levene $=1,614 ; p=0,168]$, tendo sido obtidas as respectivas médias e desvios-padrão: Norte $(M$ $=2,1 ; D P=0,38)$, Centro-Oeste $(M=2,2 ; D P=0,33)$, Nordeste $(M=2,2 ; D P=0,33)$, Sudeste $(M=2,2 ; D P=$ $0,31)$ e $\operatorname{Sul}(M=2,2 ; D P=0,32)$. Quanto à área do conhecimento, embora a ANOVA tenha detectado significância $[F(7,2142)=2,179 ; p=0,033$; teste de Levene $=0,818$; $p=0,573]$, ao se aplicar o post-hoc Tukey, nenhuma diferença estatisticamente significativa foi observada nas comparações. Nesse caso, possivelmente o tamanho da amostra tenha inflado o valor da estatística $F$ na ANOVA, porém, as distribuições não foram distintas o suficiente para confirmar diferenças na comparação $2 \times 2$ (Tabachnick \& Fidell, 2007). Tal pressuposto se confirmou ao serem analisadas as médias e desvios-padrão de cada área, conforme mostrado em sequência: Ciências Agrárias $(M$ $=2,2 ; D P=0,34)$, Ciências Biológicas $(M=2,2 ; D P=$ $0,31)$, Ciências da Saúde $(M=2,2 ; D P=0,29)$, Ciências Exatas e da Terra $(M=2,1 ; D P=0,36)$, Ciências Humanas $(M=2,2 ; D P=0,32)$, Ciências Sociais e Aplicadas $(M$ $=2,2 ; D P=0,32)$, Engenharias $(M=2,2 ; D P=0,32) \mathrm{e}$ Linguística, Letras e Artes $(M=2,1 ; D P=0,32)$. Logo, pode-se concluir que o BES não diferiu segundo o sexo, a idade, região do país, área do conhecimento e curso dos pós-graduandos.

A correlação entre as estratégias de enfrentamento, a escala de preocupações e o BES (Tabela 1) mostrou que quase todas as relações exibiram significância estatística. Apenas duas relações não foram significativas, a saber: (a) BES versus Tempo e Recursos Financeiros; e (b) BES versus Supervisão e Desempenho (ambos fatores da escala de preocupações).

O estresse, por sua vez, apenas não se correlacionou com a Busca por Suporte Social, mas manteve relações significativas com as demais estratégias de enfrentamento e os fatores do questionário de estressores. O Foco no Problema só não se correlacionou com o fator Demandas do Curso. O Foco na Emoção e a Busca pela Religiosidade se correlacionaram com todas as variáveis. Quanto a Busca por Suporte Social, correlacionou-se somente com o BES, o Foco no Problema, o Foco na Emoção e a Busca pela Religiosidade.

Tabela 1

Índices de Correlação entre Bem-Estar Subjetivo, Estressores, Estresse e Estratégias de Enfrentamento

\begin{tabular}{ccccccccc}
\hline & 1 & 2 & 3 & 4 & 5 & 6 & 7 & 8 \\
\hline 2 & 0,029 & & & & & & & \\
3 & $-0,024$ & $0,602^{* *}$ & & & & & & \\
4 & $0,073^{*}$ & $0,395^{* *}$ & $0,422^{* *}$ & & & & & \\
5 & $-0,197^{* *}$ & $0,486^{* *}$ & $0,456^{* *}$ & $0,223^{* *}$ & & & & \\
6 & $0,472^{* *}$ & $-0,112^{* *}$ & $-0,179^{* *}$ & $-0,012$ & $-0,426^{* *}$ & & & \\
7 & $-0,082^{* *}$ & $0,287^{* *}$ & $0,307^{* *}$ & $0,183^{* *}$ & $0,550^{* *}$ & $-0,223^{* *}$ & & \\
8 & $0,125^{* *}$ & $0,115^{* *}$ & $0,119^{* *}$ & $0,145^{* *}$ & $-0,066^{*}$ & $0,123^{* *}$ & $0,162^{* *}$ & \\
9 & $0,158^{* *}$ & 0,031 & 0,019 & 0,017 & $-0,003$ & $0,219^{* *}$ & $0,090^{* *}$ & $0,283^{* *}$ \\
\hline
\end{tabular}

Notas. 1. Bem-Estar Subjetivo, 2. Tempo e Dinheiro, 3. Supervisão e Desempenho, 4. Demandas do Curso, 5. Estresse, 6. Foco no Problema, 7. Foco na Emoção, 8. Busca pela Religiosidade e/ou Pensamento Fantasioso, 9. Busca por Suporte Social.

$* p<0,05$. ** $p<0,001$. 
Santos, A. F. (2013). Um Modelo Explicativo para o Bem-Estar Subjetivo: Estudo com Mestrandos e Doutorandos no Brasil.

Teste do Modelo Explicativo do Bem-Estar Subjetivo

No desenho a ser testado (Figura 1), os estressores (correlacionados entre si) são preditores do estresse, o qual, por sua vez, prediz o uso das estratégias de enfrentamento, que, no desfecho, medeiam a produção de variabilidade do BES.

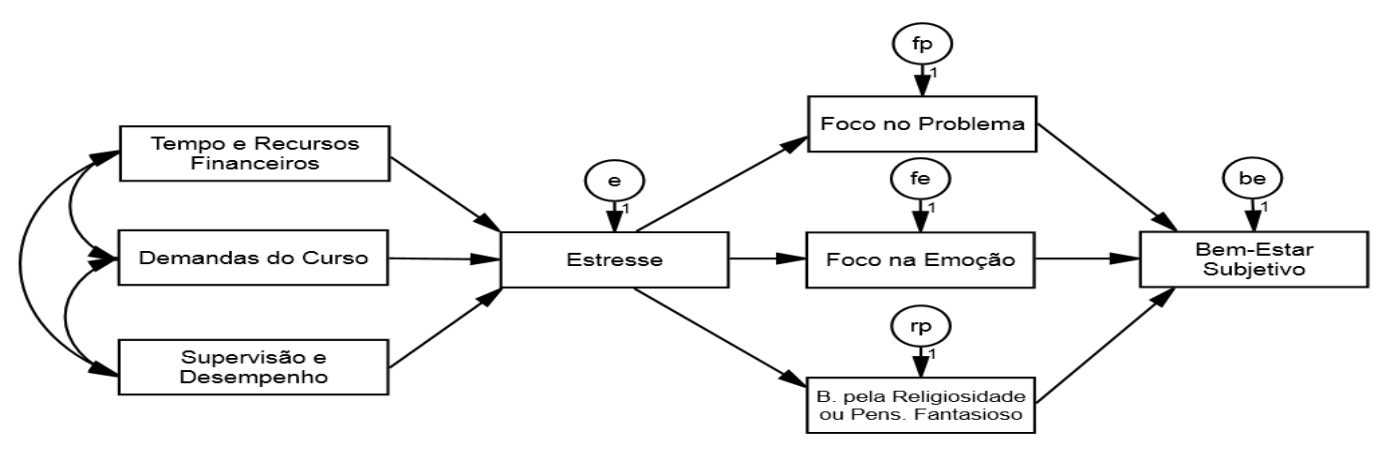

Figura 1. Modelo Inicial do Bem-Estar Subjetivo de Mestrandos e Doutorandos na Pós-Graduação.

Vale ressaltar que o modelo foi montado somente com variáveis que denotaram significância estatística na análise bivariada, considerando a relação entre antecedentes e consequentes. Por exemplo, embora o fator Tempo e Recursos Financeiros e o fator Supervisão e Desempenho não tenham exibido significância com o BES (desfecho final), eles se mostraram correlacionados ao estresse (mediador), que é a variável consequente de ambos, o que justificou a permanência na análise.

A Busca por Suporte Social foi excluída a priori do modelo, pois, ainda que tenha se relacionado com o BES, não houve relação com o estresse, que é seu antecedente imediato. Como a inclusão de variáveis nessa condição inflaria o modelo, em virtude de graus de liberdade desnecessários (Anderson \& Gerbing, 1998), e, sobretudo, contrariaria o pressuposto teórico aqui previsto (Byrne, 2010), que é o da ativação sequenciada dos estressores, estresse e enfrentamento, optou-se por excluí-la da testagem. Além disso, torna-se importante destacar que se conduziu a testagem apenas para a amostra total, visto que o sexo, a idade, a região do país, a área do conhecimento e o curso (VI's) não denotaram significância estatística em relação ao BES (principal VD), portanto, não sendo necessária, no presente estudo, a comparação de modelos explicativo segundo tais variáveis.

Dado o exposto, através da Path Analysis verificou-se a adequação do modelo e encontraram-se os seguintes índices: $\mathrm{GFI}=0,972 ; \mathrm{CFI}=0,947$; $\mathrm{ECVI}=0,129 \mathrm{e} \mathrm{RM}$ $\mathrm{SEA}=0,80 . \mathrm{Na}$ análise das regressões (setas direcionais), viu-se que 3 predições não tiveram significância estatística $(p>0,05)$, foram elas: Demandas do Curso à estresse $(\beta$ $=-0,02)$; estresse à Busca pela Religiosidade $(\beta=0,03)$; e Foco na Emoção à BES $(\beta=0,01)$.

Embora se tenha constatado um ajuste satisfatório do modelo inicial, manteve-se o princípio da parcimônia, cujo objetivo é alcançar a mais simples estrutura explicativa para o desfecho, sem a perda de confiabilidade ou qualidade do ajuste (Anderson \& Gerbing, 1998; Byrne, 2010). Com isso, um procedimento recomendado é a minimização de graus de liberdade desnecessários à modelagem, o que se fez excluindo predições não significativas. Tal ação, geralmente, melhora a adequação do modelo (Blunch, 2008; Byrne, 2010; Garson, 2003), o que foi ratificado através dos parâmetros de avaliação do modelo 2 (Figura 2): $\mathrm{GFI}=0,989 ; \mathrm{CFI}=0,979 ; \mathrm{ECVI}=$ 0,038 e RMSEA $=0,072$.

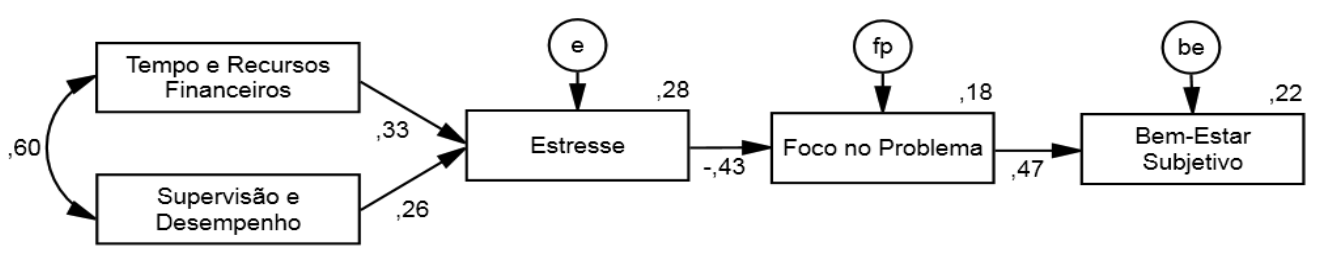

Figura 2. Modelo 2 (Final) do Bem-Estar Subjetivo de Mestrandos e Doutorandos na Pós-Graduação.

Com efeito, obtiveram-se evidências substanciais da adequação do modelo 2 após as modificações, sendo que todas as regressões agora foram significativas $(p<0,05)$.
Em suma, no modelo 2, ora denominado como modelo final, o fator Tempo e Recursos Financeiros e o fator Supervisão e Desempenho estão fortemente correlacionados 
entre si $(r=0,60)$ e predizem o estresse (respectivamente, $\beta=0,33$ e $\beta=0,26)$. Do estresse, explicou-se $28 \%$ de sua variância, predizendo negativamente $(\beta=-0,43)$ a única estratégia de enfrentamento que denota impacto efetivo sobre o BES. O Foco no Problema teve $18 \%$ de variância explicada, predizendo positivamente a variabilidade do $\operatorname{BES}(\beta=0,47)$, que foi explicada em $22 \%$.

\section{Discussão}

Este estudo teve como objetivo maior mapear as relações entre estressores, estresse, enfrentamento e BES em mestrandos e doutorandos no Brasil. Frente ao conjunto de achados da pesquisa, acredita-se que o modelo explicativo para o BES tenha produzido uma solução final satisfatória, tanto teoricamente, quanto em seus indicadores de ajuste (Blunch, 2008; Byrne, 2010). Logo, entende-se que foi alcançado êxito quanto à meta pretendida.

No que tange aos estressores, viu-se que o fator Tempo e Recursos Financeiros foi o que denotou maior impacto na produção de variabilidade do estresse. Esse fator remete a dilemas ligados à compatibilização entre a vida de pós-graduando e a vida "fora da universidade". Quanto ao tempo, o equacionamento necessário para lidar com os diversos desafios impostos pela formação e, ainda assim, manter sua rotina pessoal, revelou ser um elemento de elevada importância para a adaptação ao dia-a-dia de estudante de mestrado ou doutorado. Por ser visto, geralmente, como limítrofe na perspectiva dos formandos, isso no sentido da produção acadêmica - a exemplo do ditado do "publicar ou perecer" - ou no atendimento às inúmeras atividades pertinentes à formação - tais como cumprir disciplinas, entregar trabalhos, etc. -, percebe-se o quão relevante é a administração do tempo no processo de ajustamento e bem-estar dos pós-graduandos; achado próximo ao de outros estudos (André, 2007; Aquino, 2008; Escobar \& Verdinelli, 2010; Horta, 2006; Morosini, 2009).

No caso dos recursos financeiros, vale destacar que foi mensurado basicamente na forma de carência, não apenas para a execução da pesquisa, mas, de modo especial, pelo fato dos cursos de mestrado e doutorado ocuparem (direta ou indiretamente) boa parte do dia dos alunos, limitando, ou até impedindo, a manutenção e/ou busca de vínculos empregatícios paralelos à pós-graduação. Desde a queda nos rendimentos mensais (pela abdicação de emprego ou redução de carga horária), à política de bolsas de estudo (sem esquecer o próprio valor dessas, geralmente aquém do que se deseja), os recursos financeiros durante a formação se mostram enquanto elementos de peculiar impacto sobre a capacidade de adaptação dos estudantes, propiciando o aumento do estresse e, por consequência, redução do BES.

Ao lado do Tempo e Recursos Financeiros, o fator Supervisão e Desempenho se refere à relação aluno-orientador e a expectativa de desempenho em seu curso no papel de estressores. Isso sugere que pressões em torno da produtividade, cobranças individuais e do orientador (críticas favoráveis ou não) tendem a ser recebidas como sobrecarga no cotidiano de mestrandos e doutorandos. Embora tais aspectos sejam, em certa medida, esperados na pós-graduação, entender como se apresentam as expectativas do estudante em torno de si e do seu trabalho, e, também, analisar como se estabelece a relação aluno-orientador, parecem ser essenciais para se entender o montante de estresse causado pela busca, efetiva ou desejada, de níveis de qualidade sempre mais elevados.

No caso do fator Demandas do Curso, que trata das exigências da formação (ex. horários, aproveitamento, etc.), embora tenha apresentado significância da análise bivariada, seu impacto desapareceu no modelo final. Acredita-se que isso se deva à técnica de análise multivariada efetuada na Path Analysis, pois permite controlar o efeito da covariância de outras VI's que agem sobre a VD no modelo (Blunch, 2003; Byrne, 2010). Logo, quando mensurados simultaneamente os impactos dos estressores, tornou-se não significativa a função do fator Demandas do Curso na explicação do estresse. Em relação às estratégias de enfrentamento Busca pela Religiosidade e/ou Pensamento Fantasioso e Foco na Emoção, acredita-se que tenha ocorrido o mesmo, visto a análise simultânea desses construtos.

O estresse médio dos participantes (aproximadamente 29 pontos) ficou ligeiramente acima do ponto médio da escala (28 pontos), mostrando que, mesmo discretamente, o estresse dos pós-graduandos está acima dos $50 \%$ possíveis na pontuação da PSS. Quanto à variância explicada do estresse pelos fatores estressores, alcançou-se quase $30 \%$; um montante que pode ser considerado elevado no campo da Psicologia (Dancey \& Reidy, 2006), o que reitera a capacidade do conjunto de estressores mensurados neste estudo explicarem alterações na percepção de estresse dos mestrandos e doutorandos.

O Foco no Problema foi a único modo de enfrentamento que permaneceu no modelo, com sua ativação associada a menor estresse. Essa modalidade de enfrentamento se refere à mobilização cognitiva pautada em ações (pensamentos e comportamentos) que buscam minimizar o impacto do estressor ou mesmo extinguir o estímulo estressógeno (Lazarus \& Folkman, 1984; Skinner \& Zimmer-Gembeck, 2007). Em outras palavras, o Foco no Problema se trata de um conjunto de intenções e/ou atitudes voltadas à procura por soluções que reduzam a percepção de inadaptação e/ou mal-estar diante de uma experiência estressante (Braun-Lewensohn et al., 2009; Santos, 2010). Na literatura, vários estudos têm reiterado o efeito positivo do Foco no Problema sobre o bem-estar (Chang et al., 2007; Folkman \& Moskowitz, 2004; Taylor \& Stanton, 2007), o que foi aqui reforçado.

Quanto ao BES, obteve-se um modelo explicativo de sua variabilidade com bons índices de ajuste, além de simplicidade e plausibilidade teórica, o que permite, à primeira vista, delinear antecedentes do desfecho, ou seja, a trajetória da produção de diferenças na percepção de bem-estar; neste caso, de mestrandos e doutorandos em 
sua pós-graduação. Na presente análise foi encontrado que o BES, na relação com elementos que possam provocar decréscimo no bem-estar (estressores), é mediado pela intensidade do estresse provocado pela percepção do estressor, frente ao qual, em um segundo nível de mediação, há o uso de estratégia Foco no Problema, que visam a otimizar a adaptação no contexto avaliado.

Esse enquadramento leva a crer que o modelo elucida pontos para uma possível intervenção voltada à modificação do status do BES no ambiente dos mestrados e doutorados. Por exemplo, podem ser planejadas mudanças que reduzam o nível de sobrecarga dos fatores estressores, como também, podem ser desenvolvidas ações dirigidas ao fortalecimento do uso do Foco no Problema. Tais aspectos se mostrariam, assim prediz o modelo, como facilitadores na promoção de BES, o que, por consequência, incidiria positivamente sobre o desempenho dos estudantes e a consecução de suas metas junto à pós-graduação. Em síntese, entende-se que ações direcionadas ao BES dos mestrandos e doutorandos não impactariam apenas no aperfeiçoamento do plano acadêmico individual e institucional, mas, sobretudo, repercutiriam sobre a saúde desses alunos (Costa \& Pereira, 2007; Diener, 2000; Galinha \& Ribeiro, 2005; Siqueira \& Padovan, 2008), criando um ambiente mais favorável à criatividade e desenvolvimento de novos conhecimentos (Alencar, 2002; ME \& CAPES, 2010; Santos \& Alves, 2007); alvos precípuos do sistema de pós-graduação.

Julga-se pertinente destacar que embora existam evidências na literatura sobre BES que indiquem diferenças em relação ao sexo, idade e papéis sociais (Costa \& Pereira, 2007; Diener, 2000; Galinha \& Ribeiro, 2005; Guedea et al., 2006; Siqueira \& Padovan, 2008) e, como testado aqui, entre região do país e área do conhecimento, no presente estudo não foram confirmadas tais expectativas. Desse modo, a construção do modelo se voltou a uma perspectiva mais ampla da determinação do BES, contribuindo, assim, para a formação de um panorama generalista para explicação desse construto. De qualquer forma, cabe lembrar que uma limitação desta pesquisa foi o fato de que outras importantes variáveis sociodemográficas e profissionais não foram utilizadas para análise do desfecho, por exemplo, tempo de formação, renda individual e familiar, estado civil e pretensão de continuar na carreira acadêmica, tornando-se sugestões para comparação em investigações futuras.

Quanto aos construtos, constatou-se que o modelo funcionou bem por meio da análise conjunta dos estressores, estresse e enfrentamento. No entanto, esse desenho não anula a possibilidade de outros construtos serem inseridos para aperfeiçoamento do modelo em próximas pesquisas. Como sugestão, julga-se pertinente a verificação do impacto de variáveis como auto-estima, ansiedade e depressão (traço e/ou estado), locus de controle, valores humanos e tipologias personológicas no funcionamento explicativo do BES, a fim de expandir a compreensão em torno de sua determinação, já que a variância explicada obtida foi de
$22 \%$, noutras palavras, ainda que tenha sido proveitosa, há $78 \%$ a serem investigados.

Como outra limitação deste estudo, a ausência dos construtos e variáveis acima citados impede inferências mais amplas em relação aos fatores que modificam a percepção de bem-estar dos estudantes. Por fim, entende-se que essas sugestões de continuidade aqui descritas podem favorecer ao amadurecimento das pesquisas na área, pois fomentam a replicação do estudo, a produção de modelos concorrentes e, também, a análise confirmatória dos achados desta pesquisa.

\section{Referências}

Albuquerque, A. S., \& Tróccoli, B. T. (2004). Desenvolvimento de uma escala de bem-estar subjetivo. Psicologia: Teoria e Pesquisa, 20(2), 153-164.

Alencar, E. M. L. S. (2002). O estímulo à criatividade em programas de pós-graduação segundo seus estudantes. Psicologia: Reflexão e Crítica, 15, 63-70.

Anderson, J. C., \& Gerbing, D. W. (1998). Structural equation modeling in practice: A review and recommended two-step approach. Psychological Bulletin, 103, 411-423.

André, M. (2007). Desafios da pós-graduação e da pesquisa sobre formação de professores. Educação \& Linguagem, 10(15), 43-59.

Antoniazzi, A. S., Dell'Aglio, D. D., \& Bandeira, D. R. (1998). O conceito de coping: Uma revisão teórica. Estudos de Psicologia (Natal), 3(2), 273-294.

Aquino, E. M. (2008). Epidemiologia e saúde coletiva no Brasil: Desafios para a formação em pesquisa. Revista Brasileira de Epidemiologia, 11(Supl. 1), 151-158.

Blunch, N. J. (2008). Introduction to structural equation modeling: Using SPSS and AMOS. Thousand Oaks, CA: Sage.

Braun-Lewensohn, O., Celestin-Westreich, S., Celestin, L., Verleye, G., Verte, D., \& Ponjaert-Kristoffersen, I. (2009). Coping styles as moderating the relationships between terrorist attacks and well-being outcomes. Journal of Adolescence, 32, 585-599.

Byrne, B. (2010). Structural equation modeling with Amos: Basic concepts, applications and programming. Mahwah, NJ: Lawrence Erlbaum.

Centro de Gestão e Estudos Estratégicos. (2010). Doutores 2010. Estudos da demografia da base técnico-cientifica brasileira. Brasília, DF: Autor.

Centro de Gestão e Estudos Estratégicos. (2011). Ciência, tecnologia e inovação para o desenvolvimento das regiões Norte e Nordeste do Brasil: Novos desafios para a política nacional de CT\&I. Brasília, DF: Autor.

Chang, E. C., Sanna, L. J., Riley, M. M., Thornburg, A. M., Zumberg, K. M., \& Edwards, M. C. (2007). Relations between problem-solving styles and psychological adjustment in young adults: Is stress a mediating variable? Personality and Individual Differences, 42, 135-144.

Cohen, S., Janick-Deverts, D., \& Miller, G. E. (2007). Psychological stress and disease. JAMA, 298, 1685-1687.

Costa, L. S. M., \& Pereira, C. A. A. (2007). Bem-estar subjetivo: Aspectos conceituais. Arquivos Brasileiros de Psicologia, 59, 72-80.

Dancey, C. P., \& Reidy, J. (2006). Estatística sem matemática para a psicologia. Porto Alegre, RS: Artmed. 
Dela Coleta, J. A., \& Dela Coleta, M. F. (2006). Felicidade, bem-estar subjetivo e comportamento acadêmico de estudantes universitários. Psicologia em Estudo, 11(3), 533-539.

Diener, E. (2000). Subjective well-being. Psychological Bulletin, 95(3), 542-575.

Dusselier, L., Dunn, B., Wang, Y., Shelley, M. C., II, \& Whalen, D. F. (2005). Personal, health, academic, and environmental predictors of stress for residence hall students. Journal of American College Health, 54, 15-24.

Escobar, M. A. R., \& Verdinelli, M. A. (2010). Percepção do discente sobre produtividade científica em um programa de pós-graduação stricto sensu. In X Colóquio Internacional sobre Gestión Universitaria en América del Sur (pp. 1-14). Mar Del Plata, Argentina: Universidade Federal de Santa Catarina.

Faria, J. B., \& Seidl, E. M. F. (2005). Religiosidade e enfrentamento em contextos de saúde e doença: Revisão de literatura. Psicologia: Reflexão e Crítica, 18(3), 381-389.

Folkman, S., \& Moskowitz, J. T. (2004). Coping: Pitfalls and promise. Annual Review of Psychology, 55, 745-774.

Galinha, I., \& Ribeiro, J. L. P. (2005). História e evolução do conceito de bem-estar subjectivo. Psicologia, Saúde \& Doenças, 6(2), 203-214.

Garson, G. D. (2003). PA 765 Statnotes: An online textbook. Retrieved September 16, 2009, from http://www2.chass.ncsu. edu/garson/pa765/statnote.htm

Giancola, J. K., Grawitch, M. J., \& Bochert, D. (2009). Dealing with the stress of college: A model for adults students. Adult Education Quarterly, 59(3), 246-263.

Guedea, M. T. D., Albuquerque, F. J. B., Tróccoli, B. T., Noriega, J. A. V., Seabra, M. A. B., \& Guedea, R. L. D. (2006). Relação do bem-estar subjetivo, estratégias de enfrentamento e apoio social em idosos. Psicologia: Reflexão e Crítica, 19(2), 301-308.

Horta, J. S. B. (2006). Avaliação da pós-graduação: Com a palavra os coordenadores de programas. Perspectiva, 24, 19-47.

Koenig, H. G. (2007). Religião, espiritualidade e Psiquiatria: Uma nova era na atenção à saúde mental. Revista de Psiquiatria Clínica, 34, 5-7.

Lazarus, R. S., \& Folkman, S. (1984). Stress, appraisal and coping. New York: Springer.

Luft, C. D. B., Sanches, S. O., Mazo, G. Z., \& Andrade, A. (2007). Versão brasileira da escala de estresse percebido: Tradução e validação para idosos. Revista de Saúde Pública, 41, 606-615.

Ministério da Ciência e Tecnologia. (2010). Plano de ação em ciência, tecnologia e inovação: Principais resultados e avanços. Brasília, DF: Autor.

Ministério da Educação, \& Coordenação de Aperfeiçoamento de Pessoal de Nível Superior. (2010). Plano nacional de pós-graduação (PNPG) 2011-2020. Brasília, DF: Ministério da Educação.

Monroe, S. M. (2008). Modern approaches to conceptualizing and measuring human life stress. Annual Review of Clinical Psychology, 4, 33-52.

Morosini, M. C. (2009). A pós-graduação no Brasil: Formação e desafios. Revista Argentina de Educación Superior, 1, 125-152.

Santos, A. F. (2010). Determinantes psicossociais da capacidade adaptativa: Um modelo teórico para o estresse (Tese de doutorado, Instituto de Psicologia, Universidade Federal da Bahia, Salvador, BA, Brasil).

Santos, A. F., \& Alves, A., Jr. (2007). Estresse e estratégias de enfrentamento em mestrandos de ciências da saúde. Psicologia: Reflexão e Crítica, 20, 107-116.
Seidl, E. M. F., Tróccoli, B. T., \& Zannon, C. M. L. C. (2001). Análise fatorial de uma medida de estratégias de enfrentamento. Psicologia: Teoria e Pesquisa, 17(3), 225-234.

Siqueira, M. M. M., \& Padovam, V. A. R. (2008). Bases teóricas do bem-estar subjetivo, bem-estar psicológico e bem-estar no trabalho. Psicologia: Teoria e Pesquisa, 24, 201-209.

Skinner, E. A., \& Zimmer-Gembeck, M. J. (2007). The development of coping. Annual Review of Psychology, 58, 119-144.

Streiner, D. L., \& Norman, G. R. (2008). Health measurement scales: A practical guide to their development and use $\left(^{\text {th }}\right.$ ed.). Oxford, UK: Oxford University Press.

Tabachnick, B. G., \& Fidell, L. S. (2007). Using multivariate statistics $\left(5^{\text {th }}\right.$ ed.). New York: Harper Collins.

Taylor, S. E., \& Stanton, A. L. (2007). Coping resources, coping processes, and mental health. Annual Review of Clinical Psychology, 3, 377-401.

Vangelist, A. L. (2009). Challenges in conceptualizing social support. Journal of Social and Personal Relationships, 26, 39-51.

Verhine, R. E. (2008). Pós-graduação no Brasil e nos Estados Unidos: Uma análise comparativa. Educação (Porto Alegre), 31(2), 166-172.

Voltarelli, J. C. (2002, out./dez.). Estresse e produtividade acadêmica [Editorial]. Medicina (Ribeirão Preto), 35(4), 451-454. 